\title{
Comparison of Three Dimensional Test and Double Disc Synergy Test for detection of Extended Spectrum $\beta$-Lactamase (ESBL) producing Gram negative bacteria
}

\author{
Biswas SM 1, Mia MRA 2, Ara N 3, Ibrahim M 4, Nasir TA 5, Yunus S 6
}

\begin{abstract}
Extended spectrum $\beta$-lactamase (ESBLs), produced by Gram negative organisms, are enzymes capable of hydrolyzing extended spectrum cephalosporins, penicillins and monobactam but inactive against cephamycin and imipenem. Detection of some ESBL strains are missed by Double disc synergy test but detected by three dimensional test. So this study was carried out to see the rate of ESBL producer by three dimensional test and double disc synergy test among Gram negative bacteria. Total 110 Gram negative isolates were studied, among them 30 were wound swab isolates and 80 were laboratory isolates, of which $88(80 \%)$ were ESBL producer. Three dimensional tests detected ESBL in $88(80 \%)$ strain whereas $66.36 \%$ strains detected by double disc synergy test, so $15(13.63 \%)$ isolates were missed by Double disc synergy test. In this study among the 30 wound swab isolates Three dimensional test detected 26 (86.67\%) whereas 14 (46.67) were detected by double disc synergy test and similarly among the 80 laboratory isolates, three dimensional test detected $62(77.5 \%)$ and double disc synergy test detected 59 (73.75\%). So, the three dimensional test has been found to be better than Double disc synergy test in the detection of ESBLs. ESBL producing organisms are resistance to most of the antibiotics but $100 \%$ sensitive to Imipenem.
\end{abstract}

\section{Key word}

ESBL, Multidrug resistance, Three dimensional test, Double disc synergy test

\section{Introduction}

The emergence and spread of drug resistance in enterobacteriaceae is a growing concern in human medicine. Resistant bacterial strains have emerged and have spread throughout the world because of the remarkable genetic plasticity of the micro-organism, heavy selective pressure of antibiotic use and the mobility of the world population. The spread of extended spectrum $\beta$-lactamases (ESBLs) producing Gram negative bacteria has become a major concern among multidrug resistant organisms. $^{1}$
ESBLs are enzymes that mediate resistance to extended-spectrum (third generation) cephalosporins (e.g., ceftazidime, cefotaxime, and ceftriaxone) and monobactams (e.g., aztreonam) but do not affect cephamycins (e.g., cefoxitin and cefotetan) or carbapenems (e.g., meropenem or imipenem). ${ }^{2}$ Majority of ESBLs producing strains are Klebsiella pneumoniae, Klebsiella oxytoca and E.coli. Other organisms reported to harbour ESBLs include Enterobacter spp, Salmonella spp, Morganella morganii, Proteus mirabilis, Serratia marcescens and Pseudomonas aeruginosa. ${ }^{3}$ ESBLs are mediated 
by plasmids and are the products of point mutations at the active site of TEM, SHV and OXA enzymes. 4

Most of the clinical diagnostic laboratories depend on traditional susceptibility tests to screen for ESBL production among Gram Negative organisms which unfortunately lacks both sensitivity and specificity to detect ESBLs. ${ }^{5}$ Another method of ESBL detection is doubledisk synergy test (DDST). The major advantage of this method is that it is technically simple. However the sensitivity of DDST may be reduced when ESBL activity is very low, leading to wide zones of inhibition around the cephalosporin and aztreonam disks, the inability of clavulanate to inhibit all ESBLs, the inability of the test to detect ESBLs in strains that also produces chromosomal cephalosporinases, and the loss of clavulanate disc potency during storage.6,7 Another disadvantage of this test is that the synergy between the amoxicillinclavulanate disc and the indicator cephalosporin may be overlooked if the inoculum is too heavy or if the discs are too far from each other. ${ }^{8}$ All these things cause diagnosis failure and mis-use of antibiotics.

Three dimentional test (TDT) is another method to detect ESBL production in gram negative bacteria. TDT gives phenotypic evidence of ESBL-induced inactivation of extendedspectrum cephalosporin or aztreonam without relying on the demonstration of inactivation of the $\beta$-lactamases by a $\beta$-lactamase inhibitor. ${ }^{7}$ Two type of Three dimensional test, direct or indirect were proposed by Thomson and Sanders. In some ESBL detection cases by direct test, the zones of inhibition are small or absent which are difficult to interpret as ESBL but these cases are detected by indirect test. If the indirect test is used, the three-dimensional test is reported to be more sensitive than the double-disk diffusion test. ${ }^{9}$ So the present study was designed to detect the ESBL production in gram negative bacteria by three-dimensional test and to compare it with the double disc synergy test and analyze antimicrobial susceptibility of the bacterial isolates.

\section{Materials and Methods \\ Settings and samples}

This study was carried out in the Microbiology \& Immunology Laboratory of Bangabandhu Sheikh Mujib Medical University (BSMMU), Dhaka during the period of July 2008 to June 2009. Total one hundred ten clinical Gram negative isolates were studied, among which eighty were isolated from different clinical samples (urine, wound swab, pus, throat swab and sputum) submitted to Microbiology \& Immunology Laboratory of BSMMU and the rest 30 were isolated from wound swabs collected from in-patient department of Burn Unit of Dhaka Medical College Hospital (DMCH).

\section{Test for determination of ESBL activity Three Dimensional Test ${ }^{7}$}

Muller Hinton Agar (MHA) plates were seeded with the inoculum of a standard sensitive strain (Escherichia.coli ATCC 25922) adjusted to MacFarland 0.5 standard. 4 wells, each $4 \mathrm{~mm}$ in diameter, were punched on inoculated agar plates. 30 $\mu \mathrm{l}$ suspension of test organism suspended in sterile distilled water with turbidity preadjusted to $5.0 \mathrm{McF}$ arland standards was poured into each well. A disc of ceftazidime, ceftriaxone, cefotaxime and aztreonam each were placed about 2 $\mathrm{mm}$ away towards the center of the plate from the wells. The plate was then incubated at $37^{\circ} \mathrm{C}$ for 16-18 hours. Heart shaped distortion of zone of inhibition around the antibiotic disc indicates ESBL production. 


\section{ORIGINAL ARTICLE}

\section{Double Disc Synergy Test ${ }^{10}$}

Mueller Hinton agar plates were seeded with standardized inoculum of the test organism (corresponding to 0.5 McFarland tube). Augmentin (20 $\mu \mathrm{g}$ amoxicillin and $10 \mu \mathrm{g}$ clavulanic acid) disc was placed in the center of the inoculated plate. Three 3rd generation cephalosporin (ceftazidime $30 \mu \mathrm{g}$, ceftriaxone $30 \mu \mathrm{g}$, cefotaxime $30 \mu \mathrm{g}$ ) and one monobactam (aztreonam $30 \mu \mathrm{g}$ ) discs were placed at $20 \mathrm{~mm}$ distance from augmentin disc. The plate was incubated overnight at $37^{\circ} \mathrm{C}$. Extension of the edge of the inhibition zone of ceftazidime, ceftriaxone, cefotaxime and aztreonam disc on the side exposed to the augmentin disc is positive for ESBL production. This extension of edge of inhibition is due to synergy of disc of Augmentin with the four discs used.

\section{Antimicrobial sensitivity test ${ }^{11}$}

All the isolates were tested for antimicrobial sensitivity using disc diffusion technique by "Kirby-Bauer method"11 against different antimicrobial agents. They included Amoxicillin

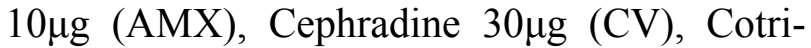
moxazole 1.25/23.75 $\mu$ g (COT), Ciprofloxacin 5 $\mu \mathrm{g}$ (CIP), Nitrofurantoin 300 $\mu$ g (NF), Nalidixic

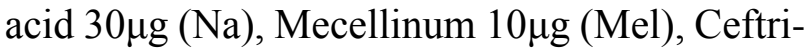
axone $30 \mu \mathrm{g}$ (CRO), Gentamicin 10 $\mu \mathrm{g}(\mathrm{CN})$, Ceftazidime $30 \mu \mathrm{g}$ (CAZ), Cefotaxime $30 \mu \mathrm{g}$

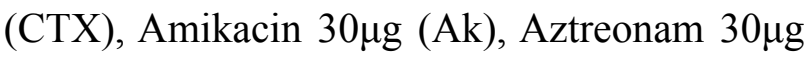

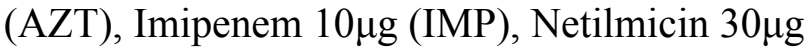
(NET). Susceptibility and resistance was determined based on the interpretative criteria recommended by the Clinical and Laboratory Standards Institute. 12 E. coli ATCC 25922 was used as the quality control strain.

\section{Result}

A total of 110 Gram negative isolates were studied, of which 30 isolates were from wound swab and 80 from laboratory isolates. Among the 110 isolates, $88(80 \%)$ were ESBL producers. Out of 30 Gram negative organisms isolated from wound swabs from Burn Unit of Dhaka Medical Collage Hospital (DMCH), 26 (83.33\%) were ESBL producer and among the 80 BSMMU laboratory isolates, $62(77.5 \%)$ were ESBL producers. The difference in ESBL production between wound swab isolates and laboratory isolates were not significant.

Table 1: ESBL producer among the Gram negative isolates studied $(n=110)$.

\begin{tabular}{|l|c|c|}
\hline Source of isolates & $\begin{array}{c}\text { No. of isolates } \\
\text { studied }\end{array}$ & $\begin{array}{c}\text { No. of ESBL } \\
\text { positive isolates (\%) }\end{array}$ \\
\hline Wound swab isolates & $\mathbf{3 0}$ & $\mathbf{2 6 ( 8 3 . 3 3 )}$ \\
\hline Clinical isolates from BSMMU Lab & $\mathbf{8 0}$ & $\mathbf{6 2}(\mathbf{7 7 . 5 )}$ \\
\hline Total & $\mathbf{1 1 0}$ & $\mathbf{8 8 ~ ( 8 0 )}$ \\
\hline
\end{tabular}


Out of 61 E. coli isolates, 51(83.61\%) were ESBL producers, among the 24 Pseudomonas spp, 16 (66.67\%) were ESBL producer and among the 14 Klebsiella spp, 12 (85.71\%) were
ESBL producers. Among 5 Enterobacter spp, 4 Acinatobacter spp and in 2 Proteus spp ESBL producer were 4 (80\%), 4 (100\%) and 1 (50\%) respectively (Fig1).

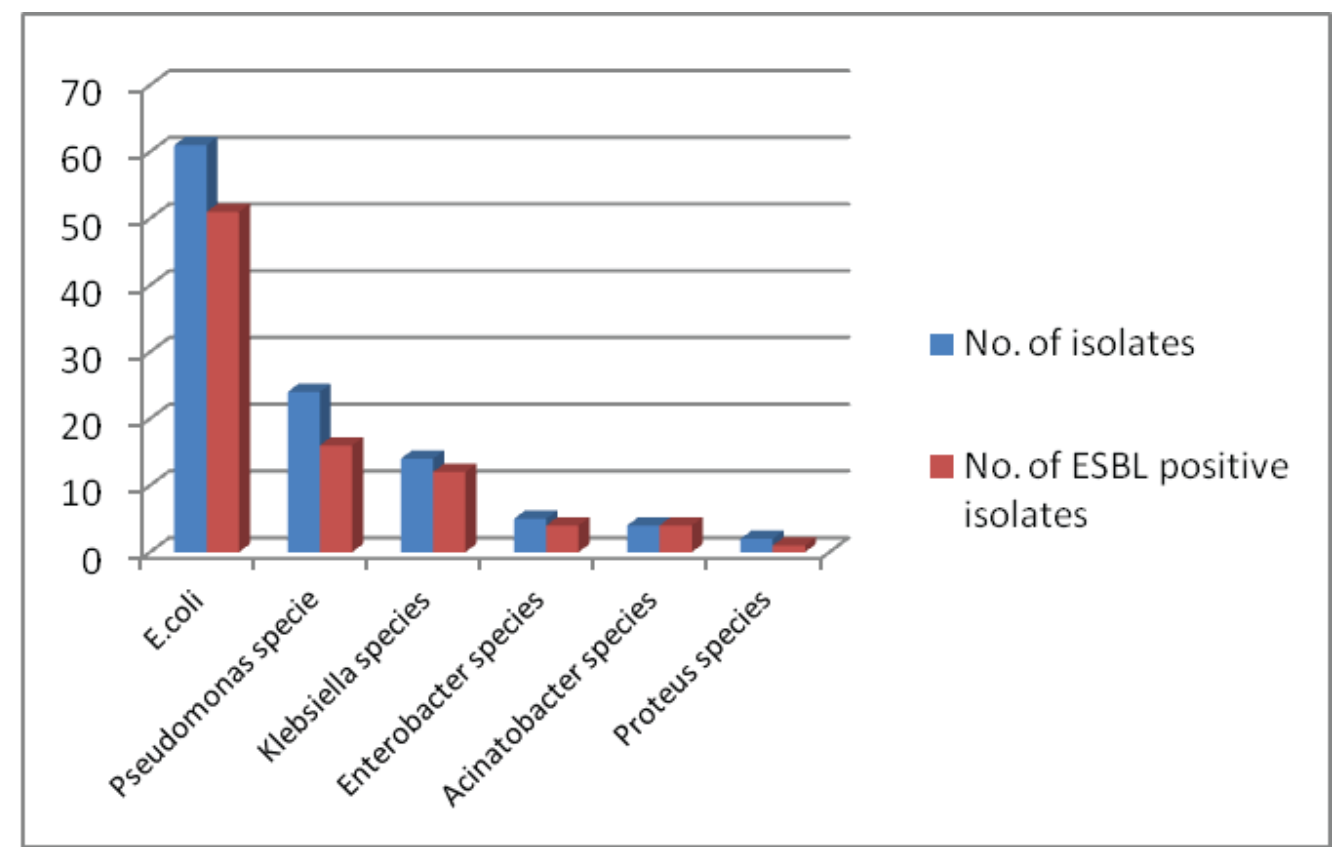

Fig 1: Distribution of different bacterial species among Gram negative bacteria studied and their ESBLs production $(n=110)$.

Among all isolates, 88 (80\%) isolates showed ESBL production by Three Dimensional Test and $73(66.36 \%)$ isolates show positivity by Double disc synergy test. 2 (3.28\%) isolates of E.coli, 2 (14.29\%) isolates of Klebsiella spp, 10 (41.67\%) isolates of Pseudomonas spp and 1 (50\%) isolate of Proteus spp were positive by three dimensional test but negative by Double disc synergy test.
No isolate that was found to be negative by three dimensional test was positive by Double disc synergy test (Table 2). The difference in ESBL detection by Three dimensional test and Double disc synergy test were significant $(p<0.05)$. Most of the ESBL producing organisms are $100 \%$ resistance to amoxicillin, cephradine, ceftazidime, ceftriaxone, aztreonam, nalidixic acid but $100 \%$ sensitive to Imipenem. 


\section{ORIGINAL ARTICLE}

Table 2: Detection of ESBL production by three dimensional test (TDT) and double disc synergy test (DDS) among the all strains studied (n=110).

\begin{tabular}{|l|c|c|c|c|}
\hline Bacterial strains (n) & $\begin{array}{l}\text { No. of 3-D test } \\
\text { positive (\%) }\end{array}$ & $\begin{array}{l}\text { No. of DDS test } \\
\text { positive (\%) }\end{array}$ & $\begin{array}{c}\text { 3-D positive but } \\
\text { DDS negative (\%) }\end{array}$ & $\begin{array}{l}\text { 3-D negative but } \\
\text { DDS positive (\%) }\end{array}$ \\
\hline E.coli (61) & $51(83.61)$ & $49(80.32)$ & $2(3.28)$ & Nil (0) \\
\hline $\begin{array}{l}\text { Klebsiella } \\
\text { species(14) }\end{array}$ & $12(85.71)$ & $10(71.42)$ & $2(14.29)$ & Nil (0) \\
\hline $\begin{array}{l}\text { Pseudomonas } \\
\text { species (24) }\end{array}$ & $16(66.67)$ & $6(25)$ & $10(41.67)$ & Nil (0) \\
\hline $\begin{array}{l}\text { Enterobacter } \\
\text { species (5) }\end{array}$ & $4(80)$ & $4(80)$ & Nil (0) & Nil (0) \\
\hline $\begin{array}{l}\text { Acinatobacter } \\
\text { species (4) }\end{array}$ & $4(100)$ & $4(100)$ & Nil (0) & Nil (0) \\
\hline Proteus species (2) & $1(50)$ & $0(0)$ & $1(50)$ & Nil (0) \\
\hline Total=110 & $88(80)$ & $73(66.36)$ & $15(13.63)$ & Nil (0) \\
\hline
\end{tabular}

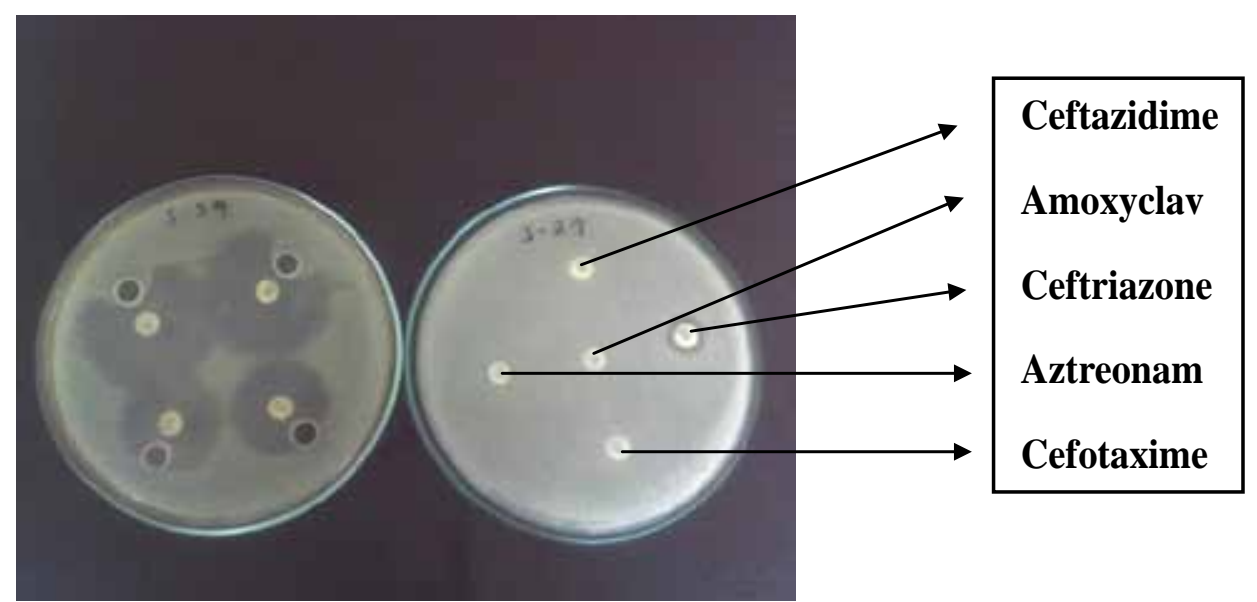

Fig 2: Three dimensional test positive but double disc synergy test negative for ESBL

(Pseudomonas spp)

\section{Discussion}

Extended-spectrum beta-lactamases (ESBLs) They are commonly expressed by constitute a growing class of plasmid-mediated Enterobacteriaceae but the species of organisms ß-lactamases which confer resistance to broad producing these enzymes are increasing and this spectrum beta-lactam antibiotics. is a cause for great concern. 
The prevalence of ESBL producing organisms is increasing worldwide and several outbreaks have been reported. ${ }^{13}$

In addition to increasing resistance to cephalosporins, resistance to other commonly used antibiotics such as fluoroquinolones is increasing. Clinical microbiology laboratories play a vital role in the detection and control of ESBL-producing Gram-negative bacilli. However, many laboratories are not fully aware of the importance of ESBL producing organisms and how best to detect them. In Europe it is estimated that $35 \%$ of ESBL producing organisms are incorrectly reported as susceptible to cephalosporins. ESBLs demonstrate low-level resistance in vitro. Routine disc susceptibility tests performed by laboratories may therefore fail to detect ESBL positive strains because these strains can be interpreted as sensitive to the extended spectrum cephalosporins. ${ }^{1}$ The traditional susceptibility methods lack sensitivity and/or specificity and this issue has prompted the search for an accurate test to detect the presence of ESBLs. ${ }^{14}$ Significant number of ESBL is also missed by Double disc synergy test; for this some authors recommended Three dimensional test for detection of ESBL producing strains. ${ }^{7,15}$ In this study a total of 110 Gram negative isolates were studied, of which 30 isolates from wound swab and 80 were laboratory isolates collected from different clinical samples. Among them $80 \%$ of the isolates were found ESBL producer. A study carried out in BSMMU by Rahman (2007) who found, ESBL in 30.90\% strains of the $\mathrm{Gm}$ negative bacteria. It is important to note that percentage of ESBL producing bacteria has been increased from $30 \%$ to $80 \%$ in last 2 yrs among Gram negative bacteria in Bangladesh. The reason of this higher percentage of ESBL in present study may be due to indiscriminate use of 3rd generation cephalosporin. Extensive use of 3rd generation cephalosporin has contributed to the evolution of ESBL. ${ }^{4}$ Other reasons include improper implementation of infection control practices and lack of implementation of antibiotic policy properly.

Among the 110 isolates, 61 were E.coli, 24 Pseudomonas spp, 14 Klebsiella spp, 5 Enterobacter spp, 4 Acinatobacter spp and 2 Proteus spp and their rate of ESBL positivity was 51 (83.61\%), 16 (66.67\%), 12 (85.71\%), 4 (80\%), 4 (100\%) and 1 (50\%) respectively. Rahman (2007) in BSMMU showed ESBL producer in Ecoli in 35.38\% strain, Klebsiella spp in $43.47 \%$, Enterobacter spp in $31.25 \%$, Proteus spp 27.11\%, Acinatobacter spp 26.32\% and Pseudomonas spp in $17.07 \%$ strains. The reason of such high rates of ESBL production in all the strains in this study might be due to overall higher rate of ESBL producer in the study isolates.

In the present study, TDT was found to be better that DDST in the detection of ESBL producing bacteria. Among the total 110 isolates ESBLs producer was found in 88 (80\%) isolates by Three dimensional method and 73 (66.36\%) by Double disc synergy method. So, 15 (13.63\%) ESBL producing isolates were missed by Double disc synergy method. The difference in ESBL detection by Three dimensional test and Double disc synergy test was significant $(p<0.05)$. The reason of such missing might be due to the coexistence of both ESBLs and plasmid-mediated Amp C $\beta$-lactamases when the Amp C $\beta$-lactamases is stably overproduced. Plasmid-mediated Amp C $\beta$-lactamases mask the synergistic effect of the clavulanic acid and the cephalosporin against ESBLs and may thus lead to false negative ESBL results. In order to detect ESBLs in the presence of Amp C $\beta$-lactamases, the use of fourth generation cephalosporins such as cefepime is required. ${ }^{16}$ The low sensitivity of DDST may also be due to the inability of clavulanate to inhibit the ESBLs variant like IRT, CMT ${ }^{17}$, GES-2. ${ }^{18}$ Moreover the penicillin-inhibitor combination, amoxicillin/clavulanic acid (AMC) is not active in vitro against a significant proportion of 
ESBLs producers. ${ }^{19}$ In a study in India Menon et al.from India reported 85.7\% ESBL positivity by three dimensional test and $14.2 \%$ positivity by double disc synergy test. ${ }^{15}$ Similar results were also reported by Datta et al in 2004 from Chandigarh, India and Thomson and Sanders in 1992 from Omaha, Nebraska. ${ }^{14,} 7$

In the present study drug resistance of all ESBLs producer to most of the antibiotics (cephalosporin, aztreonam, cotrimoxazole, gentamycin, ciprofloxacin, amikacin) were found higher. This implies that ESBL producing organisms are multidrug resistant as genes that code for ESBL are linked to other resistant genes. ${ }^{20}$ ESBL producing isolates were $100 \%$ sensitive to imipenem. According to CDC (1999), ESBLs are defined as enzymes which hydrolyze 3rd generation cephalosporins but sensitive to imipenem.

Screening for ESBL production needs to be carried out routinely in every clinical diagnostic laboratory to guide clinicians in proper selection of antibiotics. Continued monitoring of the susceptibility pattern of ESBL producing bacteria will provide invaluable information in proper clinical management.

\section{References}

1. Svard L. Evaluation of phenotypic and genotypic extended-spectrum beta-lactamase detection method. Uppsala Universitet. 2007;73-77.

2. CDC. Laboratory Detection of Extended Spectrum beta-lactamases (ESBLs), 1999. http://www.cdc.gov/ncidod/hip.

3. Thomson KS. Controversies about extended-spectrum and Amp C $\beta$-lactamases. Emerging Infectious Disease. 2001;7(2):333-336.

4. Jacoby GA, Medeiros AA. More extended spectrum beta-lactamases. Antimicrobial Agents Chemotherapy. 1991;35:1697-1704.

5. Bradford PA. Extended-spectrum $\beta$-lactamases in the 21st century: characterization, epidemiology and detection of this important resistance threat. Clinical Microbiology Review. 2001;14(4):933-951.

6. Paterson DL, Bonomo RA. Extended-spectrum $\beta$-lactamases: a clinical update. Clinical Microbiology Review. 2005;18:657-686.

7. Thomson KS, Sanders CC. Detection of ESBLs in members of the family enterobacteriaceae:

comparison of the double disc and three-dimensional test. Antimicrobial Agents and Chemotherapy. 1992;36:1877-1882.

8. Coudron PE, Moland SE, Sanders CC. Occurrence and detection of extended-spectrum $\beta$-lactamases in members of the family enterobacteriaceae at veterans medical center: seek and you may find. J Clin Microbiol. 1997;35:2593-7.

9. Vercauteren E, Descheemaeker P, Leven M, Sanders CC, Goossens H. Comparison of screening methods for detection of ESBL and their prevalence among blood isolates of e. coli and klebsiella spp. in a Belgian teaching hospital. Journal of Clinical Microbiology. 1997;35:2191-2197.

10. Jarlir V, Nicolas MH, Fournier G, Philippon A. ESBLs conferring transferable resistance to newer $\beta$-lactam agents in enterobacteriaceae: hospital prevalence and susceptibility patterns.

Review Infectious Disease. 1988;10:867-878.

11. Baur AW, Kirby WMM, Sherris JC and Turck. Antibiotic susceptibility testing by a standardized single disc method. The American Journal of Clinical Pathology. 1966;36:493-496.

12. Performance Standards for Antimicrobial Susceptibility Testing; Sixteenth Informational

Supplement. 2006, M100-S16, Clinical and Laboratory Standards Institute, Wayne, PA.

13. Al-Jasser AM. Extended-Spectrum $\beta$-lactamases (ESBLs): a global problem. Kuwait Medical Journal. 2006;38:171-185.

14. Datta P, Thakur A, Mishra B, Gupta V. Prevalence of clinical strains resistant to various

$\beta$-lactamas in a tertiary care hospital in India. Indian J Med Res, 2004;57:146-9.

15. Menon T, Bindu D, Kumar CPG, Nalini S, Thirunarayan MA. Comparison of double disc and 


\section{Comparison of Three Dimensional Test and Double Disc Synergy Test}

three dimensional methods to screen for ESBL producers in a tertiary care hospital. Brief communications. 2006;24:117-120.

16. Essack SY. Laboratory detection of extended spectrum beta-lactamases (ESBLs) - the need for a reliable, reproducible method. Diagnostic Microbiology and Infectious Disease. 2000;37:293-295.

17. Canton R, Morosini MI, Martin O, Maza S and Gomez EG. IRT and CMT $\beta$-lactamases and inhibitor resistance. Clinical Microbiol Infect. 2008;14(1):53-62.

18. Weldhagen GF, Poirel L and Nordmann P. Ambler class a extended-spectrum $\beta$-lactamases in pseudomonas aeruginosa: novel development and clinical impact. Antimicrobial Agents and Chemotherapy. 2003;47(8):2385-2392.

19. Stratchounski L, Edelstein I, Narezkina A, Edelstein M, Pimkin M. In vitro activity of cefoperazone/sulbactam vs amoxicillin/clavulanic acid and piperacillin/tazobactam against extended spectrum $\beta$-lactamase (ESBL)-producing strains of escherichia coli and klebsiella pneumoniae. 2006. E-mail:website@antibiotic.ru.

20. Ahmed I, Salam A. Extended spectrum $\beta$-lactamases and bacterial resistance. Pakistan Journal of Medical Science. 2002;18(2):151-155. 